\title{
A High-Density Seismic Network for Earthquake Early Warning in Taiwan Based on Low Cost Sensors
}

\section{by Yih-Min Wu, Da-Yi Chen, Ting-Li Lin, Chih-Yih Hsieh, Tai-Lin Chin, Wen-Yen Chang, Wei-Sen Li, and Shaw-Hsung Ker}

\section{INTRODUCTION}

Because of persistent collisions between the Philippine Sea plate and the Eurasian plate, Taiwan has been constantly threatened by large and devastating earthquakes that often cause large losses of life and property. To reduce losses caused by future damaging earthquakes, it is crucial for Taiwan to seek solutions through scientific research. The Earthquake Early Warning System (EEWS) is one of the most promising tools for alleviating threats caused by large earthquakes, and has been tested and operated in many countries (Allen et al., 2009; Lee and Wu, 2009; Satriano et al., 2011). Taiwan has been developing an EEWS and is one of the leading countries in EEWS practices (Wu et al., 1998, 1999, 2011; Wu and Teng, 2002; Hsiao et al., 2009, 2011).

The present EEWS in Taiwan has been operated by the Central Weather Bureau (CWB) since 1995 and consists of 109 telemetered seismic stations that span the entire region of Taiwan. The EEWS can provide earthquake information within $20 \mathrm{~s}$ following an earthquake occurrence (Hsiao et al., 2009, 2011; Wu and Teng, 2002). Although a 20 s reporting time is short, if the number of seismic stations operating within the network is increased, this time period can still be reduced. However, the cost of building such a high-density seismic network by traditional, force-balance seismometers is extremely high.

Since the 1990s, the Micro Electro Mechanical Systems (MEMS) accelerometers introduced in seismic applications (Holland, 2003) have been cost-saving miniature devices and ideal for recording strong ground motions. The Earthquake Early Warning (EEW) research group at National Taiwan University (NTU) worked with a technology corporation to develop a $P$-wave alert device named Palert (Fig. 1) that uses MEMS accelerometers for onsite earthquake early warning. The cost of the Palert device is less than $1 / 10$ the cost of traditional strong-motion instruments and can record real-time, three-component acceleration signals. The Palert signal resolution is 16 bits with a $-2 g$ to $+2 g$ range and the sampling rate is 100 samples per second. Once an earthquake is detected by the trigger algorithm embedded in a Palert, a corresponding earthquake watch or warning alarm is sent by the system. The trigger algorithm includes procedures for the continuous monitoring of peak ground acceleration (PGA), the short-termaverage (STA)/long-term-average (LTA) ratio (Allen, 1978), and the peak amplitude of the filtered (a high pass at the $0.075 \mathrm{~Hz}$ corner) vertical displacement of the $P$ wave $(P d$; Wu and Kanamori, 2005a, 2008a,b; Wu et al., 2007). Palert also has networking capabilities that include the streaming of real-time acceleration signals to the data collection server, the automatic connection of up to two servers, and the synchronization of clocks by the network time protocol (NTP). Therefore, Palert devices can be connected in order to build a regional EEWS (Kanamori, 2005; Wu and Kanamori, 2005b). An experimental system was successfully tested in Hualien, Taiwan (Wu and Lin, 2013). Because of this successful experiment for both onsite and regional EEWS purposes, we began to install Palert devices over the entire island of Taiwan. In this work, we present the concept and the results from a high-density low-cost sensor network that consisted of $400 \mathrm{~Pa}$ lert devices deployed for both onsite and regional EEWS in Taiwan during the testing period from June 2012 to May 2013.

\section{SYSTEM CONFIGURATION}

Since 2010 and supported by the National Science Council (NSC), NTU began to build up the Palert real-time strong-motion network. Following three years of installation, 400 stations were deployed and configured. Figure 2 provides the distribution of the Palert stations. Most of the stations are located in elementary schools where power and internet connections are provided. Therefore, the cost of building the network was reduced. To provide four hours of backup power, a display for shaking intensity, and a mechanism for issuing a sound warning, a touch screen controller named $i$ Touch (Fig. 3) was installed with a Palert device. The devices were installed in elementary schools for the purposes of cost reduction and earthquake hazard-mitigation education.

The configuration of the Palert real-time strong-motion network is shown in Figure 4. Palert devices in the field continuously send signals in real time to two central stations in the NTU, and the Grid Center of the Academia Sinica for data processing and storage. For earthquake monitoring and research 


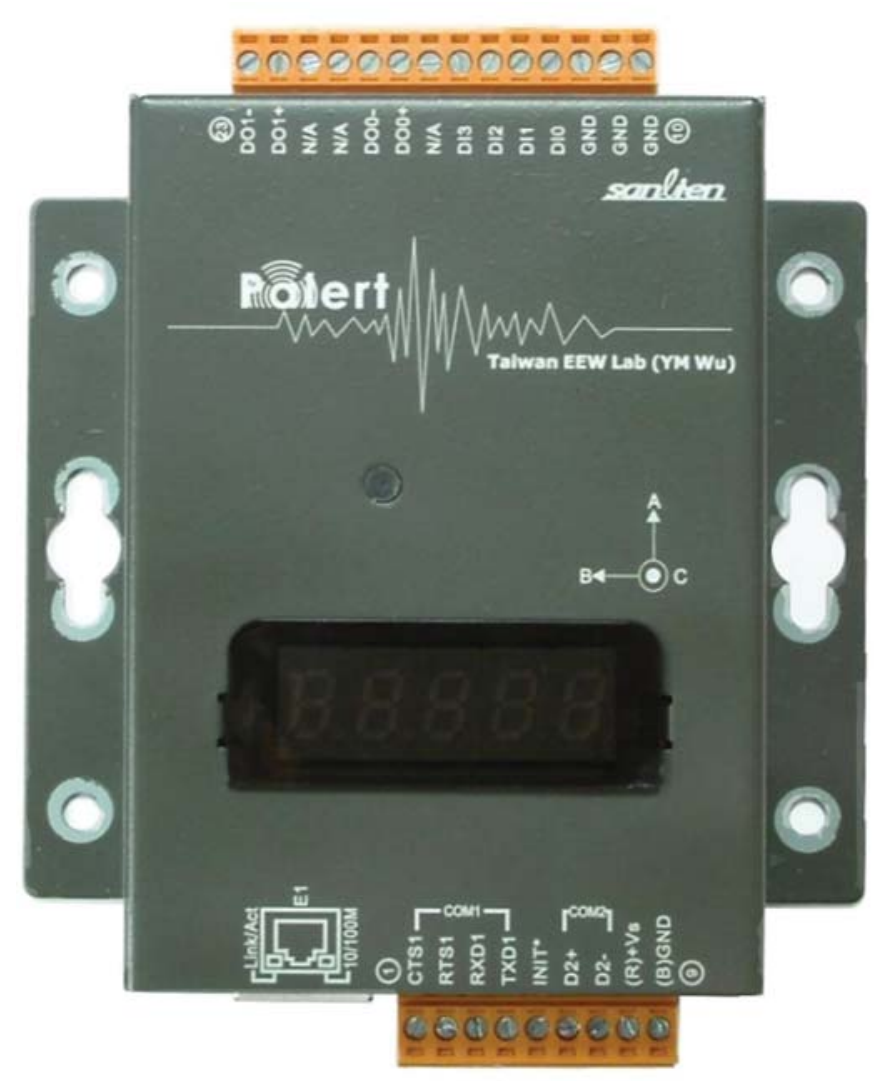

A Figure 1. The Palert Earthquake Early Warning (EEW) device.

purposes, real-time signals are also delivered to the CWB, National Cheng Kung University (NCKU), and the National Taiwan University of Science and Technology (NTUST). Figure 5 provides the signal-processing configuration of the Palert network. At the field site, real-time signals are processed by the Palert devices. Three-component acceleration signals are processed onsite for detecting $P$ arrival and continuously double integrated to displacement signals for calculating the average period, $\tau_{\mathrm{c}}$ (Kanamori, 2005; Wu and Kanamori, 2005b), and $P d$. Following the final integration, a high-pass filter at the $0.075 \mathrm{~Hz}$ corner is applied to remove low-frequency drift and to obtain the filtered displacement. Finally, the filtered displacement signals are differentiated in order to obtain the velocity signals. The real-time signals are sent to the central stations every second simultaneously via the internet. The information includes three-component acceleration and filtered vertical-displacement waveforms $1 \mathrm{~s}$ in length. When an earthquake occurs, the Palert system automatically detects $P$-wave arrival, and once the $P d$ or PGA are larger than $0.35 \mathrm{~cm}$ or 80 gal, respectively, the Palert system begins its alert with a warning sound. The $P$-wave information including the $P$ wave's first arrival, $\tau_{c}$, the peak amplitude of the acceleration $(P a)$, the velocity $(P v)$, and the displacement (i.e., $P d)$ for the initial three seconds of $P$ waves are also sent to the central stations through the TCP/IP connections. Tasks of the central stations include data clustering and processing. For real-time seismic data processing, real-time signals are processed and

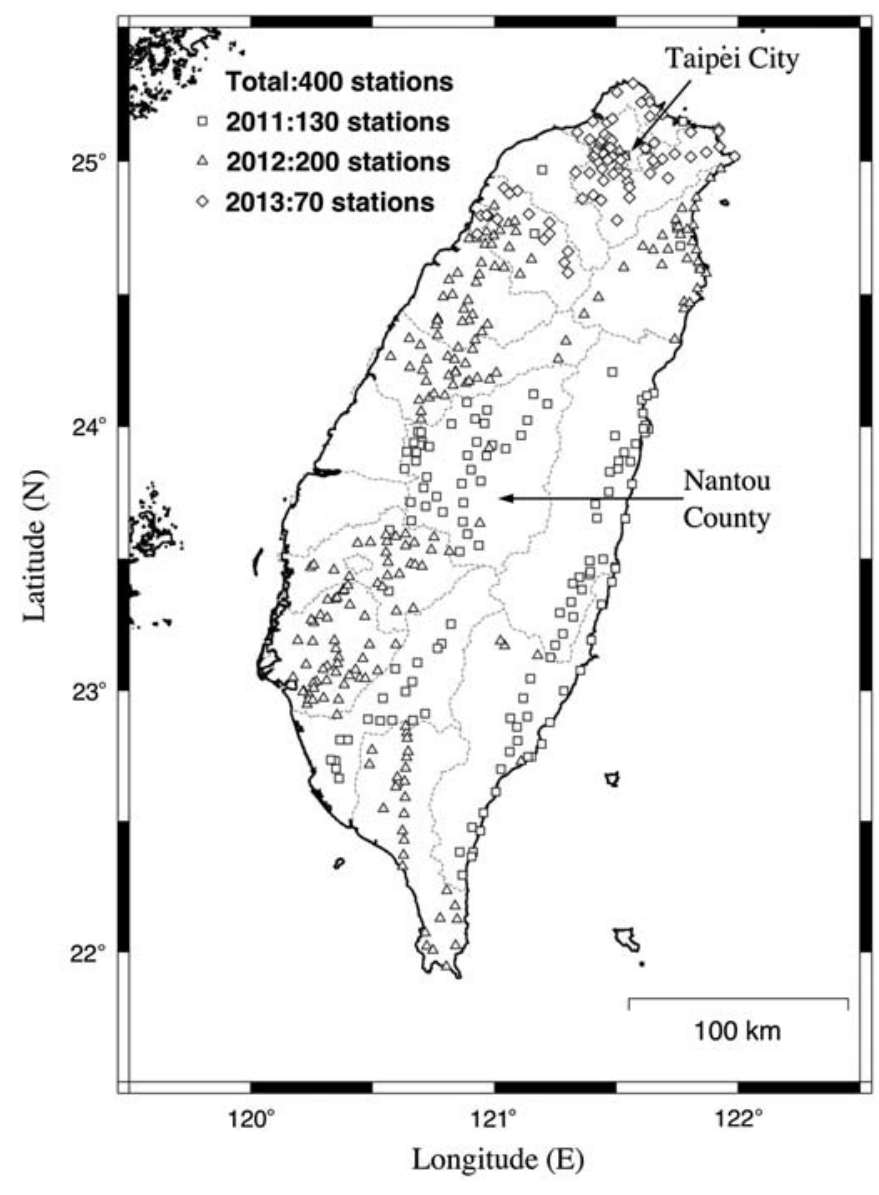

$\Delta$ Figure 2. The station distribution of the Palert EEW system.

stored within the Earthworm system developed by the U.S. Geological Survey (USGS). In the Earthworm system, signals are again processed in order to detect the arrival of the $P$ wave (Hsiao et al., 2011; Chen et al., 2012). P-wave information detected by the Earthworm platform is also sent to a shared memory. During the final stage, once eight Palert stations are triggered, an event is declared. The TcPd.c program fetches the event parameters stored within the shared memory and computes the EEW information. The hypocentral location is determined using a traditional earthquake location algorithm with a half-space linear increasing-velocity model (Wu and Lin, 2013). The magnitude determination is based on the relationship of $P d\left(M_{P d}\right.$; Wu and Zhao, 2006) and $P v\left(M_{P v}\right)$ attenuation, and hypocentral distance. Within the Palert system, for events with magnitudes less than 6.0, we noticed that the uncertainty of $M_{P v}$ was smaller than $M_{P d}$ due to a low signalto-noise ratio. Therefore, for events with an $M_{P v}$ less than 6.0, $M_{P v}$ was utilized for magnitude determination. In contrast, for events with an $M_{P v}$ larger than 6.0, $M_{P d}$ was employed. For magnitude determination, the $\tau_{\mathrm{c}}$ parameter is also processed by the TcPd.c program. Because Palert is a low-gain instrument with a relatively low signal-to-noise ratio in $\tau_{c}$ determinations for moderate and small events, we currently do not use magnitude information from the $\tau_{\mathrm{c}}$ parameter within the Palert network. 


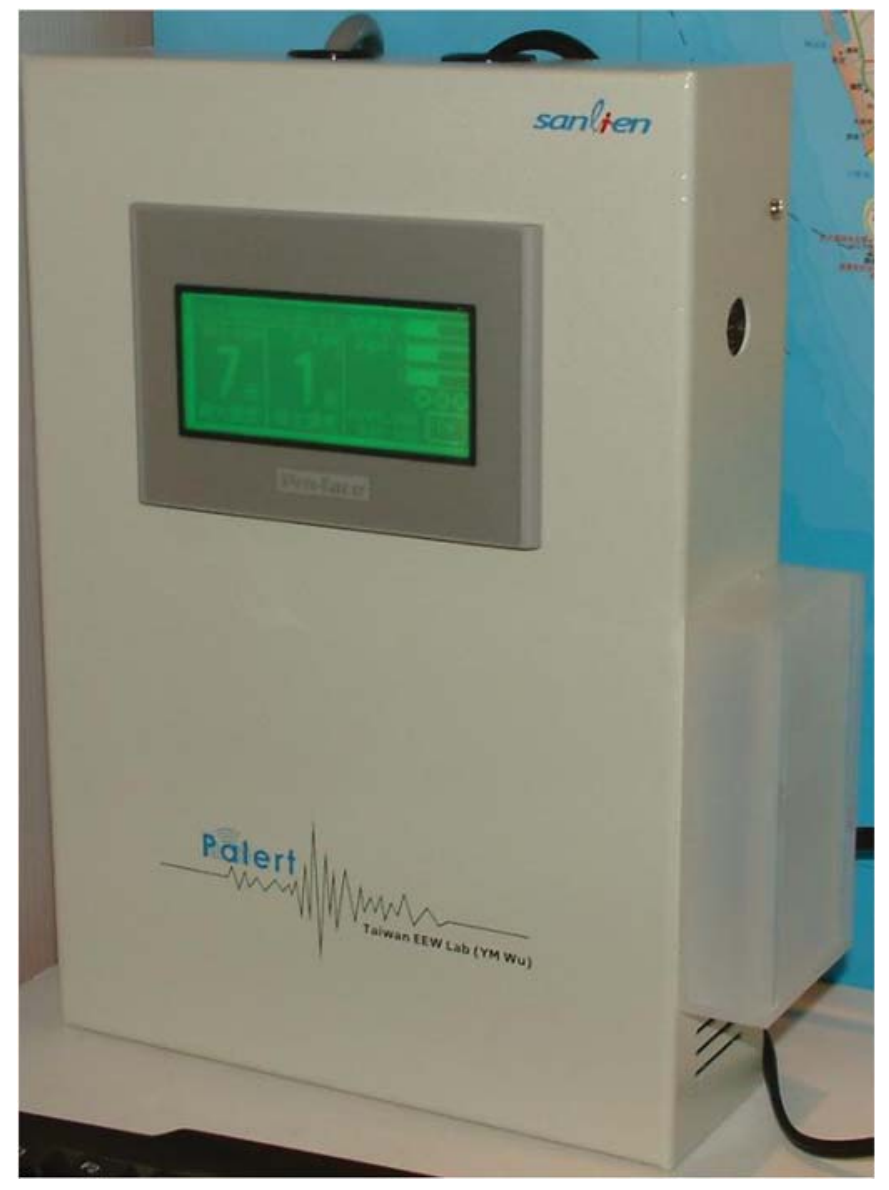

$\Delta$ Figure 3. The ITouch device is installed with a Palert device in order to provide backup power, a display, and a sound warning.

\section{Palert System Configuration}

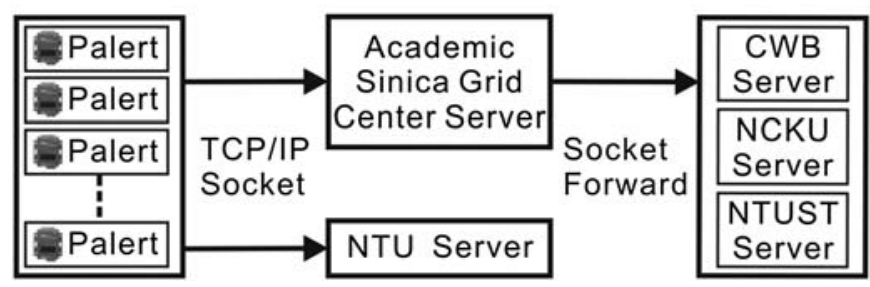

A Figure 4. The system configuration of the Palert network.

\section{SYSTEM PERFORMANCE}

Since June 2012, the implemented EEWS has operated using the Palert network. During the period from June 2012 to May 2013, 27 earthquakes with $M_{\mathrm{L}}$ magnitudes ranging from 4.6 to 6.1 have been detected. The signals were well recorded by the central stations. In general, $80 \%$ of local stations routinely and reliably send data to the central stations. Power interruptions and internet firewalls at some sites are the reason that $20 \%$ of local stations cannot send data to central stations. Figure 6 provides strong motion records for the 27 March $2013 M_{\mathrm{L}} 6.1$ Nantou earthquake. Thus far, this earthquake is the largest
Palert Signals Processing Configuration

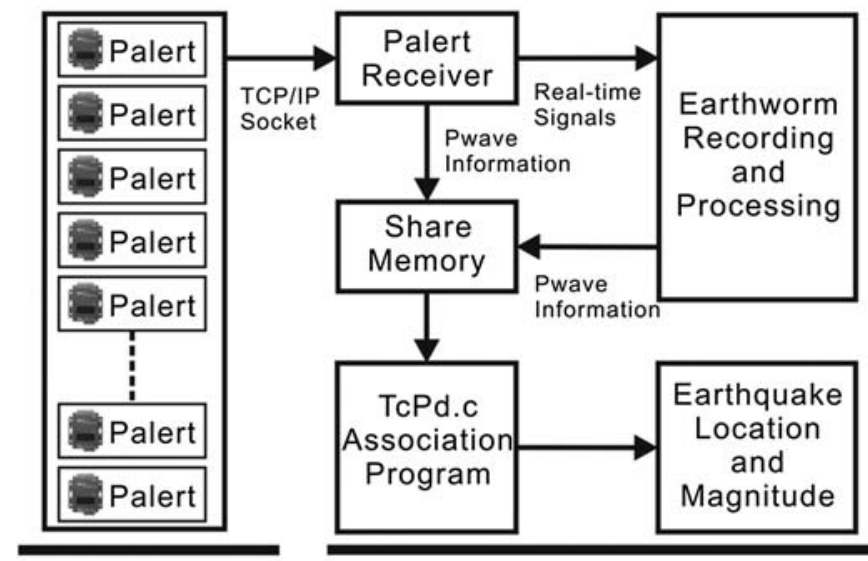

Field Station On-site Warning

\section{Central Station Regional Warning}

A Figure 5. The signal-processing configuration of the Palert network.

EW-component, Earthquake 2013/03/27 02:03:20

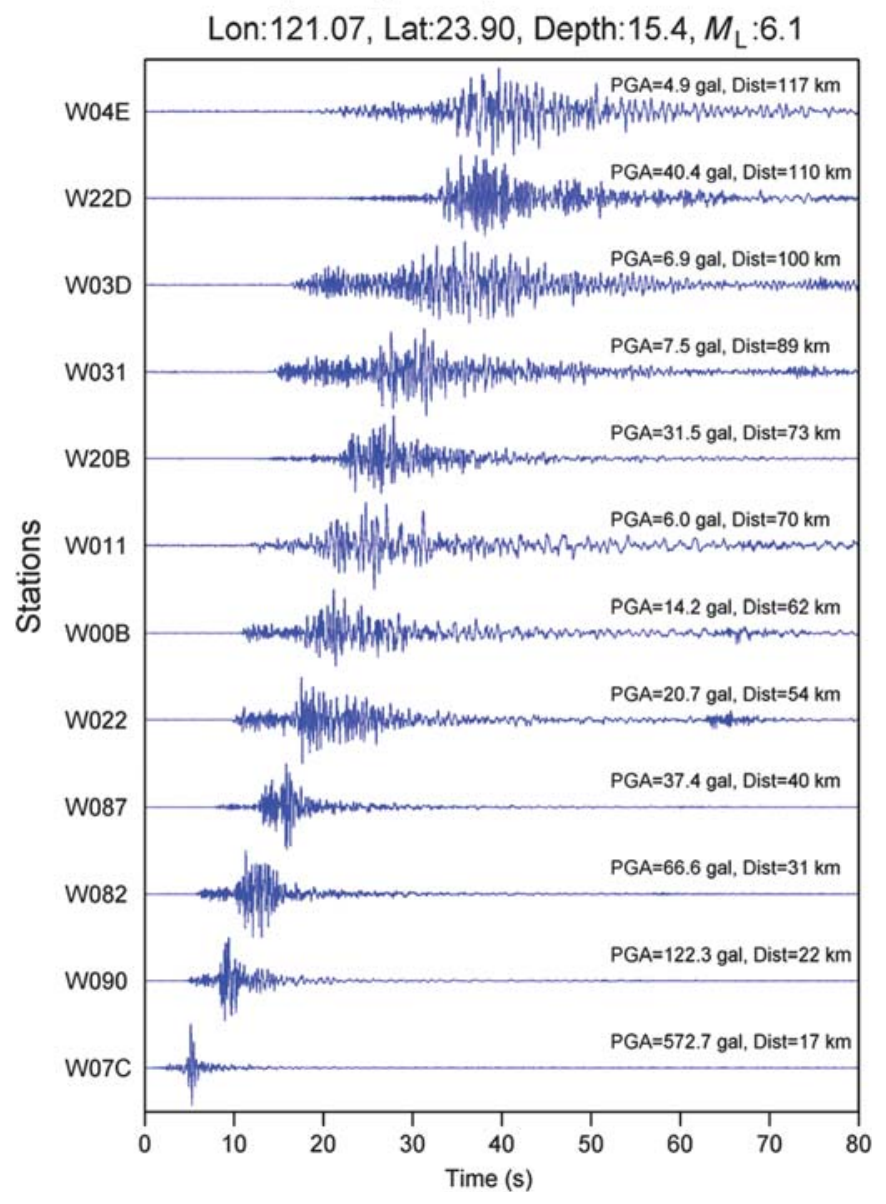

A Figure 6. Strong-motion records of the 27 March $2013 M_{\mathrm{L}} 6.1$, Nantou earthquake as recorded by one of the central stations of the Palert network. 
event that has been detected by the Palert network. EEWS performance of the Palert system is summarized in Figure 7. The online real-time results of the Palert network were compared with those reported by the CWB, determined off-line and manually (http://www.cwb.gov.tw, last accessed May 2013). The initial warning alarm is triggered by the first eight $P$ alert stations that detect the $P$ wave and ignite other reactions in an early stage. However, the Palert system will continue to collect $P$ wave information from local stations and improve the accuracy of the estimated hypocenter and magnitude until the end of an earthquake for a shaking-map determination. The real-time results shown in Figure 7 are the initial triggering results.

Hypocenters provided by the Palert network versus those from the CWB catalogue are plotted in Figure 7. The hypocenters determined by the two systems agree, with the exception of a few offshore events that have larger differences. The average difference in epicenter location was $15.8 \mathrm{~km}$ with a standard deviation of $16.1 \mathrm{~km}$. The average difference in focal depth was $0.9 \mathrm{~km}$ with a standard deviation of $19.0 \mathrm{~km}$. Prior to 2013, due to a fewer number of stations installed and a lack of $S$-arrival times, the large location errors present in the Palert system were expected. However, after 2013, with more stations installed in Taiwan, the quality of the location estimations improved over those from 2012.

The magnitudes $\left(M_{P}\right)$ determined by the Palert network and the corresponding $M_{\mathrm{L}}$ values from the CWB earthquake catalog are listed. Figure 7 also provides a plot of $M_{\mathrm{P}}$ values against those of the CWB earthquake catalog. In general, magnitude uncertainty was on the order of 0.3 , an acceptable value for EEWS.

For the year of Palert EEWS network operation we achieved an early warning earthquake reporting time $\operatorname{Tr}$ the time between earthquake occurrence and the time the EEWS provides warning information, varying from 9 to $28 \mathrm{~s}$, with an
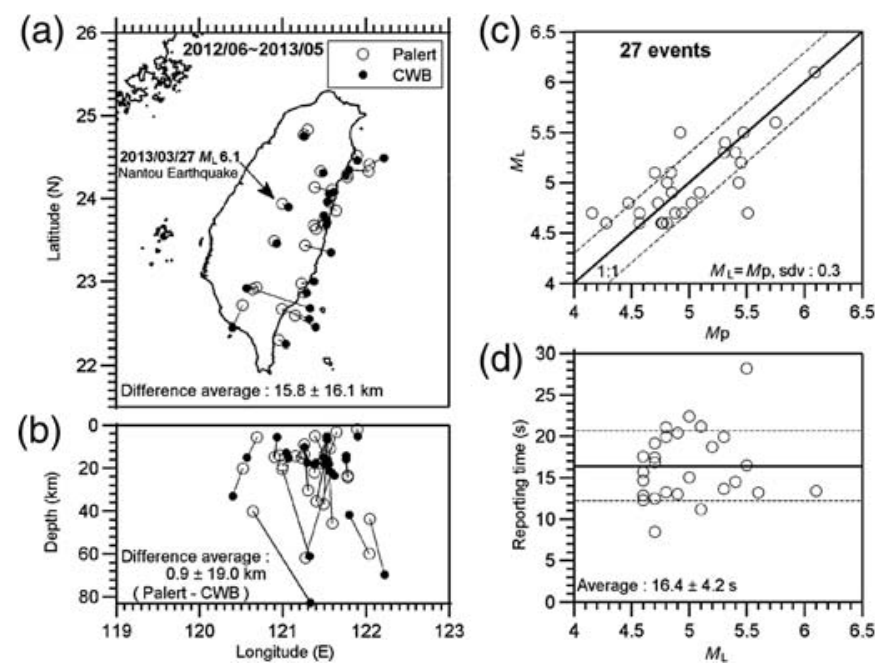

A Figure 7. A comparison of earthquake information, as follows: (a) epicenters, (b) focal depths, and (c) the magnitudes given by the real-time Palert network and the CWB published earthquake catalogs. (d) The reporting times of the Palert network. average of $16.4 \mathrm{~s}$ and a standard deviation of $4.2 \mathrm{~s}$ (Fig. 7). The results indicated that for the majority of events the Palert EEWS can issue an early warning report within approximately $20 \mathrm{~s}$ following an earthquake occurrence. Practical regional EEW is feasible using the Palert system.

\section{DISCUSSION}

The reporting time for the Palert system is similar to that of the CWB EEWS, which consists of conventional strong motion instruments (Wu et al., 1999; Wu and Teng, 2002; Hsiao et al., 2009). The system offers early warning to regions with an epicentral distance larger than $50 \mathrm{~km}$. Additionally, Palert also has an onsite alert function that triggers a warning sound once the $P d$ exceeds $0.35 \mathrm{~cm}$ or the PGA is larger than 80 gal (Wu et al., 2011). The major advantage of the onsite $P d$ approach is that it can provide a valuable early warning for epicentral regions. Figure 8 provides the resulting early warning leading times near epicentral regions for the 27 March 2013 Nantou $M_{\mathrm{L}} 6.1$ earthquake. The early warning leading time is defined as the interval between the time when the filtered vertical displacement exceeds $0.35 \mathrm{~cm}$ and the time when the PGA is larger than $80 \mathrm{gal}$ (Wu et al., 2011). There are two stations for which the time of the filtered vertical displacement exceeding $0.35 \mathrm{~cm}$ is later than the time that a PGA larger than 80 gal is recorded. However, the majority of stations have a 1-2 s lead time. It is worth noting is that the station (W07C) that recorded the largest shaking (PGA 572 gal) had a lead time of $2.7 \mathrm{~s}$, a sufficient time for automatic EEWS to make an emergency safety response.

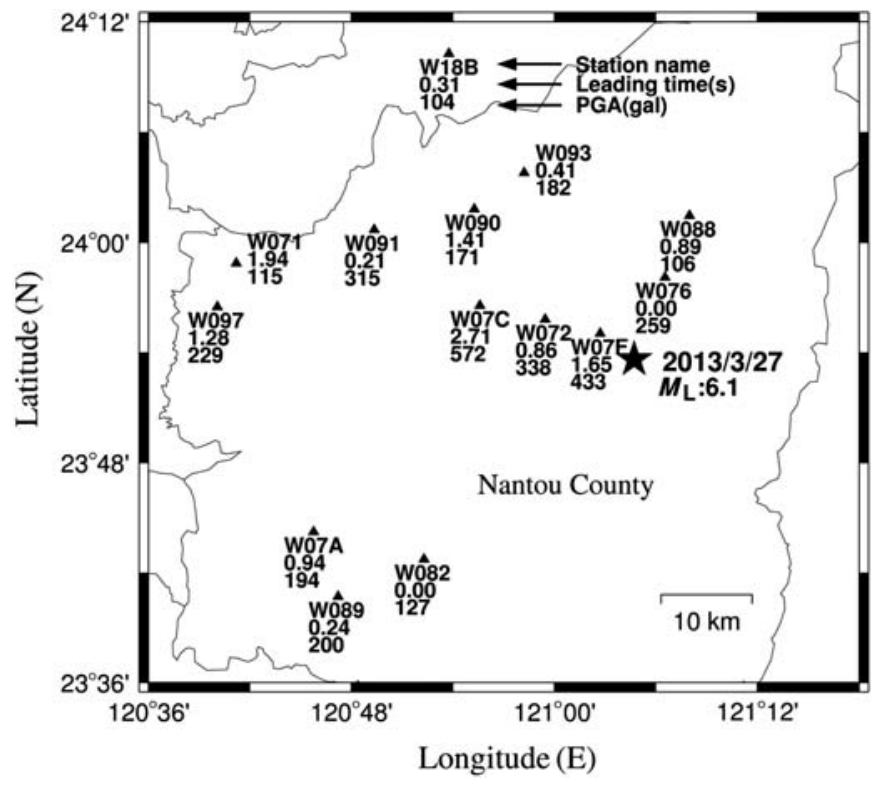

A Figure 8. The early warning lead times for Palert stations close to the epicentral region of the 27 March 2013 Nantou $M_{\mathrm{L}} 6.1$ earthquake. The leading time is defined as the interval between the time when the filtered vertical displacement exceeds $0.35 \mathrm{~cm}$ and the time for a PGA larger than 80 gal. 
Nantou Earthquake 2013/03/27 02:03:20 ML 6.1
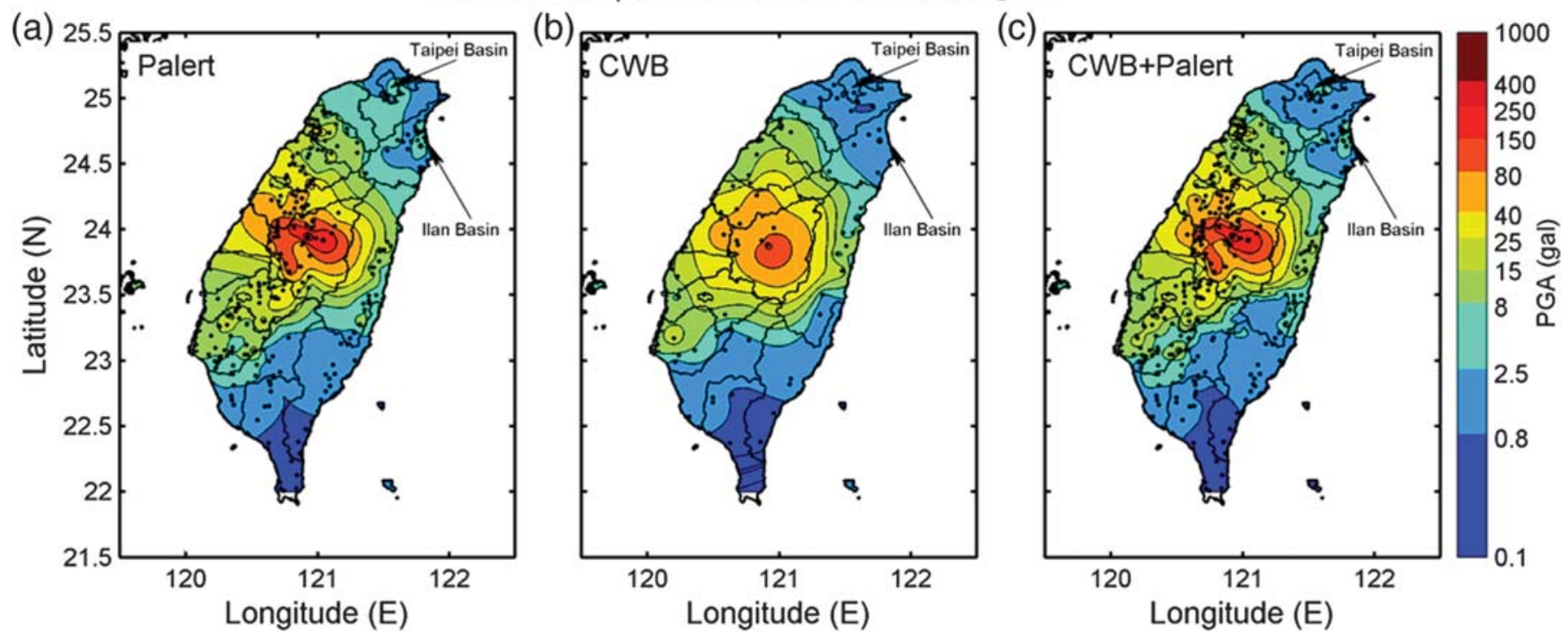

$\Delta$ Figure 9. The shaking maps determined by (a) the Palert, (b) the CWB, and (c) the CWB + Palert stations.

The present EEWS in Taiwan consists of 109 seismic stations. The Palert network has almost four times this number of stations. For earthquake rapid reporting purposes (Wu et al., 1997, 2000), the Palert network can provide a detailed shaking map for damage assessments. Figure 9 provides the shaking maps for the 27 March 2013 Nantou $M_{\mathrm{L}} 6.1$ earthquake obtained from the CWB and Palert networks. Obviously, the Palert network provides a more detailed shaking condition. For example, the Ilan and Taipei basins could easily be identified, suggesting basin effects. Palert signals were also sent to the CWB. Therefore, for a detailed shaking map (Fig. 9c) for emergency response purposes, the $\mathrm{CWB}$ can combine signals from the two networks. Here, the reader should note that number of Palert stations shown in Figure 9a is fewer than that in Figure 2. The major reasons include the following: (1) most of the stations close to Taipei City were built after the 2013 Nantou $M_{\mathrm{L}} 6.1$ earthquake, (2) $20 \%$ of the local stations cannot send data to the central stations mentioned above, and (3) large shakings during this earthquake may cause the loss of $10 \%$ of signals. Ten percent of local stations cannot send data to the central stations, as generally occurred after $S$ waves. We suggest that this phenomenon may be caused by the short instability of both power and internet during large shakings.

In the near future, at least 100 new stations will be deployed, and the Palert network will become even denser. With such a high station density, obtaining an accurate and near real-time observed-shaking map is feasible. Therefore, groundshaking propagation can be described in detail, which is helpful either when dispatching emergency response personnel and services or tracing earthquake rupturing. For example, the center of the initially triggered stations can be regarded as an initial rupture center, similar to the effective epicenter (Teng et al., 1997). The high-shaking cover area could be converted to an effective magnitude (Teng et al., 1997; Lin and Wu, 2010; Lin et al., 2011). Therefore, without a traditional earthquake-location process, we can still obtain earthquake information, which is crucial for an emergency response. Traditional magnitude determinations require a distance correction. Occasionally, error in earthquake location may lead to an over- or underestimation of earthquake magnitude. The concept of an effective epicenter and magnitude could be important for practical earthquake monitoring, especially for large earthquakes.

Currently, the Palert network uses the peak amplitude of $P$ waves for magnitude determinations. When a large earthquake occurs, a magnitude saturation problem may exist especially for $M_{P d}(\mathrm{Wu}$ et al., 2006). However, with such a high station density, magnitude determinations using the coverage area for a certain threshold of the PGA (Lin and Wu, 2010) and the Pd (Lin et al., 2011) could be used in the EEWS for solving the magnitude saturation problem. For a rapid reporting system, the empirical method of Wu and Teng (2004) for quick $M_{\text {w }}$ determinations using time integration over the strongshaking duration for absolute values of acceleration records could be used within the Palert system. Lin and Wu (2012) tested the system for the $2011 M_{\mathrm{w}} 9.0$ Great Tohoku Earthquake. The results indicated that this empirical approach is appropriate for extremely large events without a saturation problem and is important in damage assessments and tsunami early warnings.

\section{CONCLUSIONS}

In this paper, we describe the installation of a real-time, strongmotion network with a high station density that incorporates low-cost MEMS sensors for EEW and rapid reporting purposes, and present the performance of the Palert network in Taiwan. Currently, in Taiwan, when an earthquake occurs, earthquake information can be determined within $20 \mathrm{~s}$ with a reasonable 
uncertainty. Our results indicate that a practical regional EEWS is feasible. To detect the occurrence of an earthquake, each individual Palert device uses an onsite $P d$ approach. Based on the 27 March 2013 Nantou $M_{\mathrm{L}} 6.1$ earthquake, most stations displayed a 1-2 s lead time as compared with the traditional method using PGA as a threshold in regions close to the epicenter. Using a dense array of stations, the Palert network can provide a real-time shaking map for both EEW and rapid reporting applications.

Because of its low cost, the Palert device may have commercial potential. The EEWS can be established for relatively little expense, or Palert sensors can be readily added to an existing seismic network in order to increase the density of the network. Thus far, Palert devices have been installed in China, Indonesia, Mexico, and Taiwan. To test the feasibility of providing EEW to New Delhi, India, a pilot Palert network is scheduled to be installed near the Himalayan region. Results from the Palert network in Taiwan are encouraging for the further utilization of EEWS in other countries. $\mathbf{Y}$

\section{ACKNOWLEDGMENTS}

This research was supported by the Central Weather Bureau (CWB) and the National Science Council. The authors also wish to thank M. Boese for reviewing this article and providing many constructive comments. The GMT software from Wessel and Smith (1998) was used in plotting part of the figures and is gratefully acknowledged.

\section{REFERENCES}

Allen, R. V. (1978). Automatic earthquake recognition and timing from single traces, Bull. Seismol. Soc. Am. 68, 1521-1532.

Allen, R. M., P. Gasparini, O. Kamigaichi, and M. Bose (2009). The status of earthquake early warning around the world: An introductory overview, Seismol. Res. Lett. 80, no. 5, 682-693, doi: 10.1785/ gssrl.80.5.682.

Chen, D. Y., T. L. Lin, Y. M. Wu, and N. C. Hsiao (2012). Testing a $P$-wave earthquake early warning system by simulating the 1999 Chi-Chi, Taiwan, $M_{\mathrm{w}} 7.6$ earthquake, Seismol. Res. Lett. 83, no. 1, 103-108, doi: $10.1785 /$ gssrl.83.1.103.

Holland, A. (2003). Earthquake data recorded by the MEMS accelerometer: Field testing in Idaho, Seismol. Res. Lett. 74, no. 1, 20-26, doi: 10.1785/gssrl.74.1.20.

Hsiao, N. C., Y. M. Wu, T. C. Shin, L. Zhao, and T. L. Teng (2009). Development of earthquake early warning system in Taiwan, Geophys. Res. Lett. 36, L00B02, doi: 10.1029/2008GL036596.

Hsiao, N. C., Y. M. Wu, L. Zhao, D. Y. Chen, W. T. Huang, K. H. Kuo, T. C. Shin, and P. L. Leu (2011). A new prototype system for earthquake early warning in Taiwan, Soil Dyn. Earthq. Eng. 31, 201-208, doi: $10.1016 /$ j.soildyn.2010.01.008.

Kanamori, H. (2005). Real-time seismology and earthquake damage mitigation, Annu. Rev. Earth Planet. Sci. 33, 195-214, doi: 10.1146/ annurev.earth.33.092203.122626.

Lee, W. H. K., and Y. M. Wu (2009). Earthquake monitoring and early warning systems, in Encyclopedia of Complexity and Systems Science, R. A. Meyers (Editor), 11 volumes, Springer, New York, 10370 pp.

Lin, T. L., and Y. M. Wu (2010). Magnitude estimation using the covered areas of strong ground motion in earthquake early warning, Geophys. Res. Lett. 37, L09301, doi: 10.1029/2010GL042797.
Lin, T. L., and Y. M. Wu (2012). A fast magnitude estimation for the M 9.02011 Great Tohoku Earthquake, Seismol. Res. Lett. 83, no. 4, 667-671, doi: 10.1785/0220110119.

Lin, T. L., Y. M. Wu, and D. Y. Chen (2011). Magnitude estimation using initial $P$-wave amplitude and its spatial distribution in earthquake early waning in Taiwan, Geophys. Res. Lett. 38, L09303, doi: 10.1029/2011GL047461.

Satriano, C., Y. M. Wu, A. Zollo, and H. Kanamori (2011). Earthquake early warning: Concepts, methods and physical grounds, Soil Dyn. Earthq. Eng. 31, no. 2, 106-118, doi: 10.1016/j.soildyn.2010.07.007.

Teng, T. L., Y. M. Wu, T. C. Shin, Y. B. Tsai, and W. H. K. Lee (1997). One minute after: Strong-motion map, effective epicenter, and effective magnitude, Bull. Seismol. Soc. Am. 87, 1209-1219.

Wessel, P., and W. H. F. Smith (1998). New improved version of Generic Mapping Tools released, Eos Trans. $A G U$ 79, no. 47, 579, doi: 10.1029/98EO00426.

Wu, Y. M., and H. Kanamori (2005a). Rapid assessment of damaging potential of earthquakes in Taiwan from the beginning of $P$ waves, Bull. Seismol. Soc. Am. 95, no. 3, 1181-1185, doi: 10.1785/ 0120040193.

Wu, Y. M., and H. Kanamori (2005b). Experiment on an onsite early warning method for the Taiwan early warning system, Bull. Seismol. Soc. Am. 95, no. 1, 347-353, doi: 10.1785/0120040097.

Wu, Y. M., and H. Kanamori (2008a). Development of an earthquake early warning system using real-time strong motion signals, Sensors 8, no. 1, 1-9, doi: 10.3390/s8010001.

Wu, Y. M., and H. Kanamori (2008b). Exploring the feasibility of on-site earthquake early warning using close-in records of the 2007 Noto Hanto earthquake, Earth Planets Space 60, 155-160.

Wu, Y. M., and T. L. Lin (2013). A test of earthquake early warning system using low cost accelerometer in Hualien, Taiwan, in Early Warning for Geological Disasters: Scientific Methods and Current Practice, F. Wenzel and J. Zschau (Editors), Springer, Berlin, Heidelberg, New York.

Wu, Y. M., and T. L. Teng (2002). A virtual sub-network approach to earthquake early warning, Bull. Seismol. Soc. Am. 92, no. 5, 20082018, doi: 10.1785/0120010217.

Wu, Y. M., and T. L. Teng (2004). Near real-time magnitude determination for large crustal earthquakes, Tectonophysics 390, nos. 1-4, 205-216, doi: 10.1016/j.tecto.2004.03.029.

Wu, Y. M., and L. Zhao (2006). Magnitude estimation using the first three seconds $P$-wave amplitude in earthquake early warning, Geophys. Res. Lett. 33, L16312, doi: 10.1029/2006GL026871.

Wu, Y. M., C. C. Chen, T. C. Shin, Y. B. Tsai, W. H. K. Lee, and T. L. Teng (1997). Taiwan rapid earthquake information release system, Seismol. Res. Lett. 68, no. 6, 931-943, doi: 10.1785/gssrl.68.6.931.

Wu, Y. M., J. K. Chung, T. C. Shin, N. C. Hsiao, Y. B. Tsai, W. H. K. Lee, and T. L. Teng (1999). Development of an integrated earthquake early warning system in Taiwan- Case for Hualien earthquakes, Terr. Atmos. Ocean. Sci. 10, 719-736.

Wu, Y. M., H. Kanamori, R. Allen, and E. Hauksson (2007). Determination of earthquake early warning parameters, $\tau_{\mathrm{c}}$ and $P_{d}$, for southern California, Geophys. J. Int. 170, no. 2, 711-717, doi: 10.1111/j.1365-246X.2007.03430.x.

Wu, Y. M., W. H. K. Lee, C. C. Chen, T. C. Shin, T. L. Teng, and Y. B. Tsai (2000). Performance of the Taiwan rapid earthquake information release system (RTD) during the 1999 Chi-Chi (Taiwan) earthquake, Seismol. Res. Lett. 71, no. 3, 338-343, doi: 10.1785/ gssrl.71.3.338.

Wu, Y. M., T. L. Lin, W. A. Chao, H. H. Huang, N. C. Hsiao, and C. H. Chang (2011). Faster short-distance earthquake early warning using continued monitoring of filtered vertical displacement: A case study for the 2010 Jiasian earthquake, Taiwan, Bull. Seismol. Soc. Am. 101, no. 2, 701-709, doi: 10.1785/0120100153.

Wu, Y. M., T. C. Shin, and Y. B. Tsai (1998). Quick and reliable determination of magnitude for seismic early warning, Bull. Seismol. Soc. Am. 88, 1254-1259. 
Wu, Y. M., H. Y. Yen, L. Zhao, B. S. Huang, and W. T. Liang (2006). Magnitude determination using initial $P$ waves: A single-station approach, Geophys. Res. Lett. 33, L05306, doi: 10.1029/ 2005 GL025395.
Yib-Min Wu Da-Yi Chen ${ }^{I}$ Chih-Yih Hsieh Department of Geosciences National Taiwan University No. 1, Sec. 4th, Roosevelt Rd. Taipei 10617, Taiwan drymwu@ntu.edu.tw yihmin.wu@gmail.com

Ting-Li Lin

Department of Earth Sciences National Cheng Kung University Tainan 70101, Taiwan
Tai-Lin Chin

Department of Computer Science and Information Engineering National Taiwan University of Science and Technology Taipei 10607, Taiwan

Wen-Yen Chang

Department of Natural Resources and Environmental Studies National Dong Hwa University Hualien 97401, Taiwan

Wei-Sen Li Shaw-Hsung Ker National Science and Technology Center for Disaster Reduction (NCDR) New Taipei City 23143, Taiwan

\footnotetext{
${ }^{1}$ Also at Central Weather Bureau, Taipei 10048, Taiwan.
} 